\title{
Emerging functional similarities and divergences between Drosophila Spargel/dPGC-1 and mammalian PGC-1 protein
}

\author{
Subhas Mukherjee ${ }^{1+}$, Mohammed A. Basar ${ }^{1}$, Claudette Davis ${ }^{1,2}$ and Atanu Duttaroy ${ }^{1 *}$ \\ Department of Biology, Howard University, Washington, DC, USA \\ ${ }^{2}$ Biology Undergraduate Program, College of Science, George Mason University, Fairfax, VA, USA
}

\section{Edited by:}

Shin Murakami, Touro

University-California, USA

Reviewed by:

Johannes Bauer, Southern

Methodist University, USA

Margarida Matos, Faculdade de

Ciencias da Universidade de Lisboa,

Portugal

\section{${ }^{*}$ Correspondence:}

Atanu Duttaroy, Department of

Biology, Howard University, 415,

College Street, NW, Washington,

DC 20059, USA

e-mail: aduttaroy@howard.edu

${ }^{\dagger}$ Present address:

Subhas Mukherjee, Department of

Pathology and Laboratory Medicine,

Winship Cancer Institute, Emory

University School of Medicine,

Atlanta, USA

\begin{abstract}
Peroxisome Proliferator Activated Receptor Gamma Co-activator-1 (PGC-1) is a well-conserved protein among all chordates. Entire Drosophila species subgroup carries a PGC-1 homolog in their genome called spargel/dPGC-1 showing very little divergence. Recent studies have reported that significant functional similarities are shared between vertebrate and invertebrate PGC-1's based on their role in mitochondrial functions and biogenesis, gluconeogenesis, and most likely in transcription and RNA processing. With the help of genetic epistasis analysis, we established that Drosophila Spargel/dPGC-1 affects cell growth process as a terminal effector in the Insulin-TOR signaling pathway. The association between Spargel/dPGC-1 and Insulin signaling could also explain its role in the aging process. Here we provided a further comparison between Spargel/dPGC-1 and PGC-1 focusing on nuclear localization, oxidative stress resistance, and a possible role of Spargel/dPGC-1 in oogenesis reminiscing the role of Spargel in reproductive aging like many Insulin signaling partners. This led us to hypothesize that the discovery of newer biological functions in Drosophila Spargel/dPGC-1 will pave the way to uncover novel functional equivalents in mammals.
\end{abstract}

Keywords: Drosophila, PGC-1, mitochondria, oogenesis, RNA processing
Homeothermic mammals utilize the Peroxisome Proliferator Activated Receptor Gamma Co-activator 1 (PGC-1) as a thermogenic regulator (maintains body temperature) to protect against excessive cold or excess calorie intake (Puigserver et al., 1998). Thus PGC-1 is expressed in tissues with high metabolic requirement and it is linked to multiple metabolic pathways such as gluconeogenesis, adipogenesis, myogenesis, and mitogenesis (Handschin and Spiegelman, 2006). Apart from metabolism, PGC-1 might play a central role in maintaining oxidative homeostasis (Austin and St-Pierre, 2012). Homologs of PGC-1 were found in all chordates including the fish genomes (Lin et al., 2005) where body temperature regulation isn't necessary (with the exception of a few marine species). Among invertebrate models, only Drosophila carries a single PGC-1 homolog in its genome (Gershman et al., 2007) whereas other invertebrate models such as yeast and $C$. elegans do not carry any PGC-1 homologous sequence (Lin et al., 2005). Drosophila PGC-1, designated as spargel/dPGC-1 (Tiefenbock et al., 2010), is well conserved in distantly related Drosophila species subgroups with its C-terminal RS and RRM domains (Figure 1A). Two notable differences between PGC-1 and Spargel/dPGC-1 are: the C-terminal FDSLL domain of PGC-1 is replaced with FEALL in all Drosophila species (Gershman et al., 2007; Tiefenbock et al., 2010; Bugger et al., 2011), and the larger size of Spargel/dPGC-1 protein as it carries $\sim 300$ more amino acids than PGC-1 (Figure 1A). Although nuclear receptors are generally known to interact with the leucine rich motifs (LXXLL) (Matsuda et al., 2004), the FEALLL variant of this motif in Drosophila is able to interact with the nuclear receptors as well (Wang et al., 2007). In light of the fact that significant functional overlap exists between the three PGC-1 homologs in mice PGC- $1 \alpha$, PGC- $1 \beta$ and PRC, which makes it difficult to tease apart their relative roles in vivo, we propose that the presence of a single Drosophila PGC-1 homolog will provide an enormous advantage to study the function of this essential transcriptional coactivator in an alternate model. Within the last few years, significant functional homologies have surfaced between mammalian PGC-1 and Drosophila Spargel/dPGC-1, which called for a discussion of this topic in greater detail.

\section{ENERGY METABOLISM}

As a transcriptional coactivator PGC-1 activates many nuclear receptor, which in turn regulate the transcription activity of variety of nuclear genes (Puigserver and Spiegelman, 2003). Similarly, Spargel/dPGC-1 regulates the expression of mitochondrial oxidative phosphorylation (OXPHOS) genes through NRF1 (Nuclear Respiratory Factor) homolog delg (Tiefenbock et al., 2010). Spargel/dPGC-1 gain of function (overexpression) correlates with an increased rate of mitochondrial oxygen consumption (Rera et al., 2011) and ATP production (Mukherjee and Duttaroy, 2013), enhanced mitochondrial DNA content, increased enzyme activity and protein production in the mitochondrial matrix (Rera et al., 2011). These observations are reminiscent of the effect of PGC- $1 \alpha$ on mitochondrial biogenesis, functional capacity and energy metabolism (Liu and Lin, 2011). Thus, regulation of 


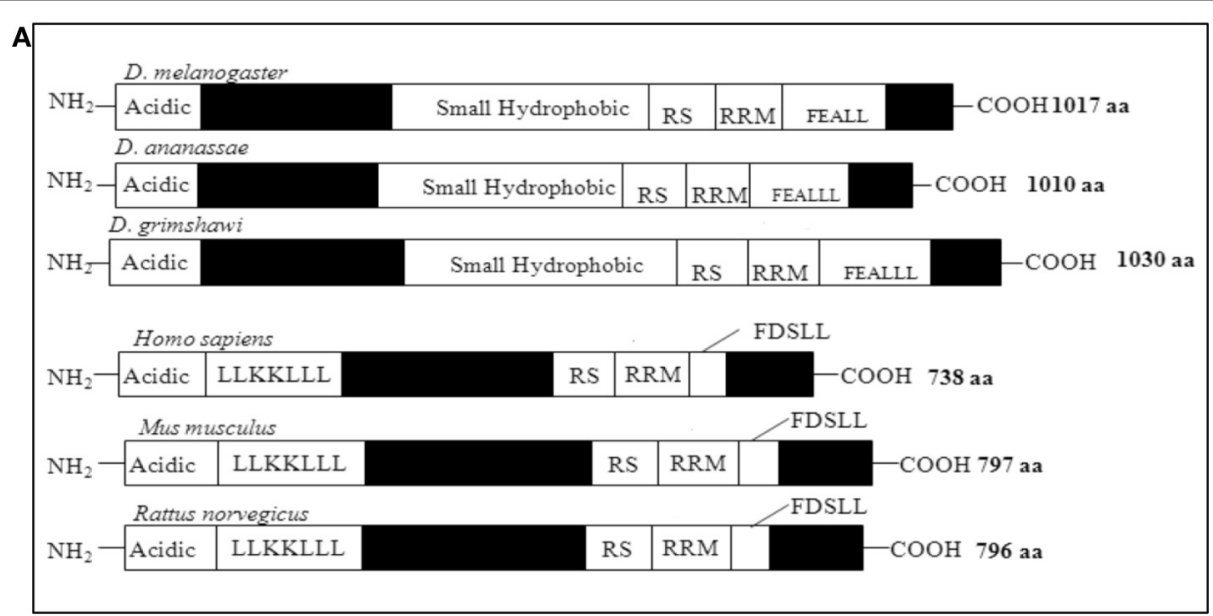

B
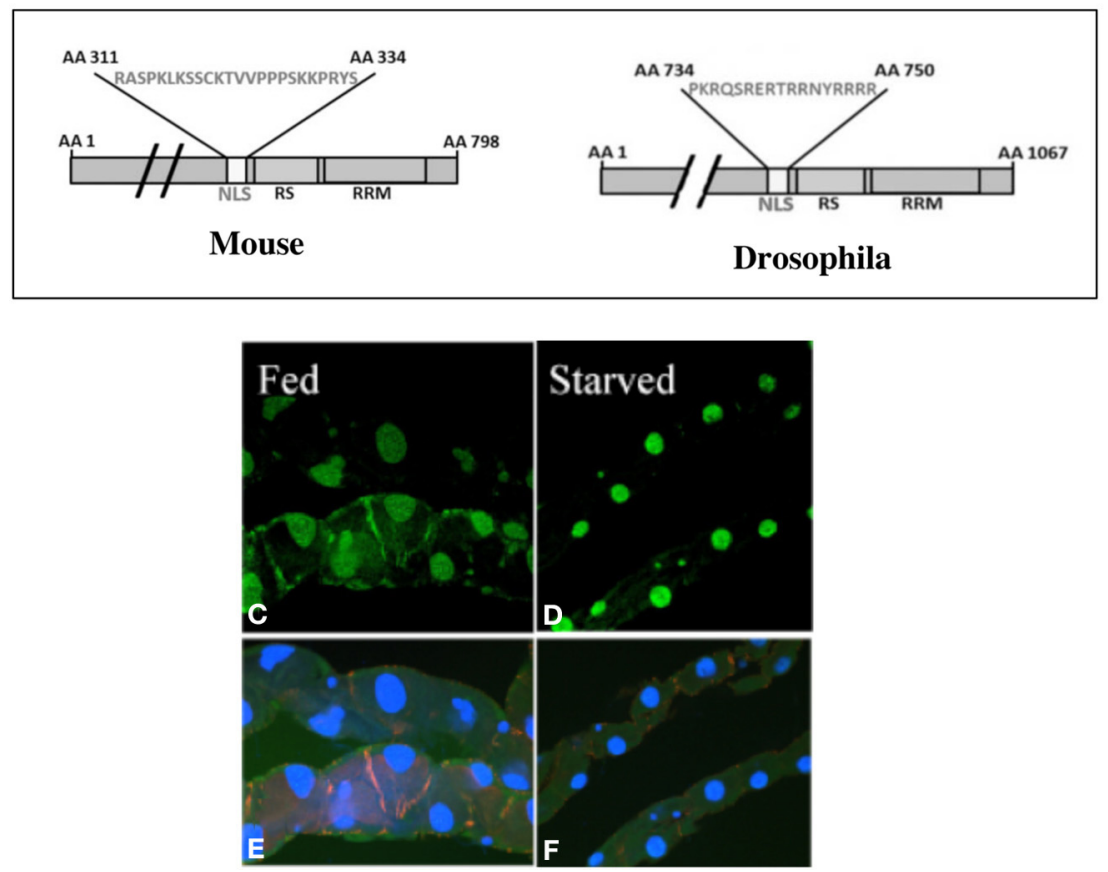

FIGURE 1 | (A) Comparison of the Spargel/dPGC-1 protein structure in 3 widely divergent subgroups of Drosophila melanogaster, D. ananassae, and

D. grimshawi (van der Linde and Houle, 2008). Vertebrate PGC-1 is about 300 amino acids shorter in length than the Spargel/dPGC-1. Filled boxes represent regions of non-homology. (B) An authentic Nuclear Localization Signal (NLS) has been found in mammalian PGC-1 with the help of NLS Predict Software. (C) Nuclear localization of Spargel is documented in the gut tissue with a Spargel-GFP protein (green). Following $48 \mathrm{~h}$ of starvation, the gut turns thinner and Insulin signaling is reduced, but it imposes no effect on nuclear localization of the Spargel-GFP protein (D). (E,F) DAPI staining of the same gut tissue. mitochondrial function is truly an ancestral function for PGC-1 group of proteins.

\section{INTRACELLULAR LOCALIZATION}

Serine-Arginine (SR) repeats and RNA Recognition Motifs (RRM) are classical hallmarks of "RNA processing domains." Furthermore, localization of PGC-1 in the nuclear compartment where it is concentrated in nuclear speckles along with splicing factor $\alpha-\mathrm{U} 1$ and SR splicing factor SC-35 are irrefutable evidence that PGC-1 is involved in the splicing complex (Monsalve et al., 2000). We recently demonstrated that a Spargel-GFP fusion protein also localizes itself in the nucleus forming distinct punctate structures (Mukherjee and Duttaroy, 2013). An authentic Nuclear Localization Signal (NLS) was uncovered in Spargel/dPGC-1 with the help of a NLS predict software (Mukherjee and Duttaroy, 2013) that most likely assists in Spargel/dPGC-1 localization into the nucleus. With the help of the same software we now located a NLS between the amino acids 311-334 in mammalian PGC-1 (score 0.93 ) (Figure 1B), which should also justify the presence of PGC-1 protein in the nucleus (Monsalve et al., 2000).

An earlier study claimed that activation of Insulin signaling is important for transport of Spargel/dPGC-1 protein from cytoplasm to the nucleus (Tiefenbock et al., 2010) and Spargel/dPGC-1 is now established as a member of the 
Insulin-TOR signaling pathway (Mukherjee and Duttaroy, 2013). Nutrient availability controls the Insulin/TOR signaling pathway from TOR downstream (Takano et al., 2001) therefore, under starvation condition, Insulin mediated signal transduction is reduced. We rationalized that if Insulin signaling is essential for nuclear localization of Spargel/dPGC-1 then reduction of this signal should sequester the protein to the cytosol. To test this prediction, gut malphigian tubule preparations were obtained from flies following two days of starvation (only water given to prevent dehydration) and analyzed for Spargel expression. Since the localization of Spargel/dPGC-1 is still restricted to the nucleus following starvation (Figures 1C-F) this supports that cellular localization of Spargel/dPGC-1 occurs independent of Insulin signaling.

\section{Spargel/dPGC-1 DOESN'T INFLUENCE ANTIOXIDANT ENZYMES}

The apparent involvement of PGC-1 in oxidative metabolism has been established from the following: PGC-1 activates Nuclear Respiratory Factor1 (NRF1); muscle specific overexpression of PGC- $1 \alpha$ induces specific antioxidants like Sod 2 and $G p X$ transcription activity (St-Pierre et al., 2006) whereas ablation of PGC- $1 \alpha$ in cultured cell cause down regulation of a whole panel of antioxidants including SOD1, SOD2, GpX, UCP1, and UCP2, resulting in hypersensitivity to hydrogen peroxide induced oxidative stress (St-Pierre et al., 2006). Another interesting observation is that cells from patients with Friedrich's Ataxia show coordinated suppression of PGC-1 and antioxidant enzymes (Coppola et al., 2009). It was inferred from these observations that PGC-1 controls mitochondrial reactive oxygen species (ROS) by regulating the antioxidant defense system (Austin and St-Pierre, 2012).

We attempted to validate this prediction in a whole animals model by utilizing the Drosophila spargel mutant hypomorph, $s r l^{1}$ (Tiefenbock et al., 2010) and a spargel transgenic line that is capable of overexpressing Spargel/dPGC-1 (Mukherjee and Duttaroy, 2013). For systemic oxidative stress generation, we used methyl violgen (commercially known as "paraquat") that is stable at room temperature. Male and female flies overexpressing Spargel were exposed to $20 \mathrm{mM}$ paraquat and both survived better than the control (Figures 2A,B). After $48 \mathrm{~h}$ of paraquat treatment, Spargel/dPGC-1 overexpression helped 50\% males to remain viable, where as in control flies 50\% survival was attained within $24 \mathrm{~h}$. Female flies appear slightly more sensitive to paraquat treatment although a significant difference still persists with respect to the control (Figures 2A,B). This increased resistance to oxidative stress in Spargel/dPGC-1 overexpressing flies makes it tempting to conclude that Spargel/dPGC1 is also involved in oxidative stress resistance and imposes the same effect on antioxidant defense enzymes like PGC-1. However, this expectation may not be true since the expression of two front line antioxidant defense enzymes SOD2 and SOD1 remain unchanged when Spargel/dPGC-1 is overexpressed (gain of function) or reduced in $s \mathrm{rl}^{1}$ hypomorphs (Figure 2C). Since these experiments were performed on whole animals as opposed to cultured cells, it might be necessary to check the status of these antioxidant enzymes in PGC-1KO mice. Secondly,

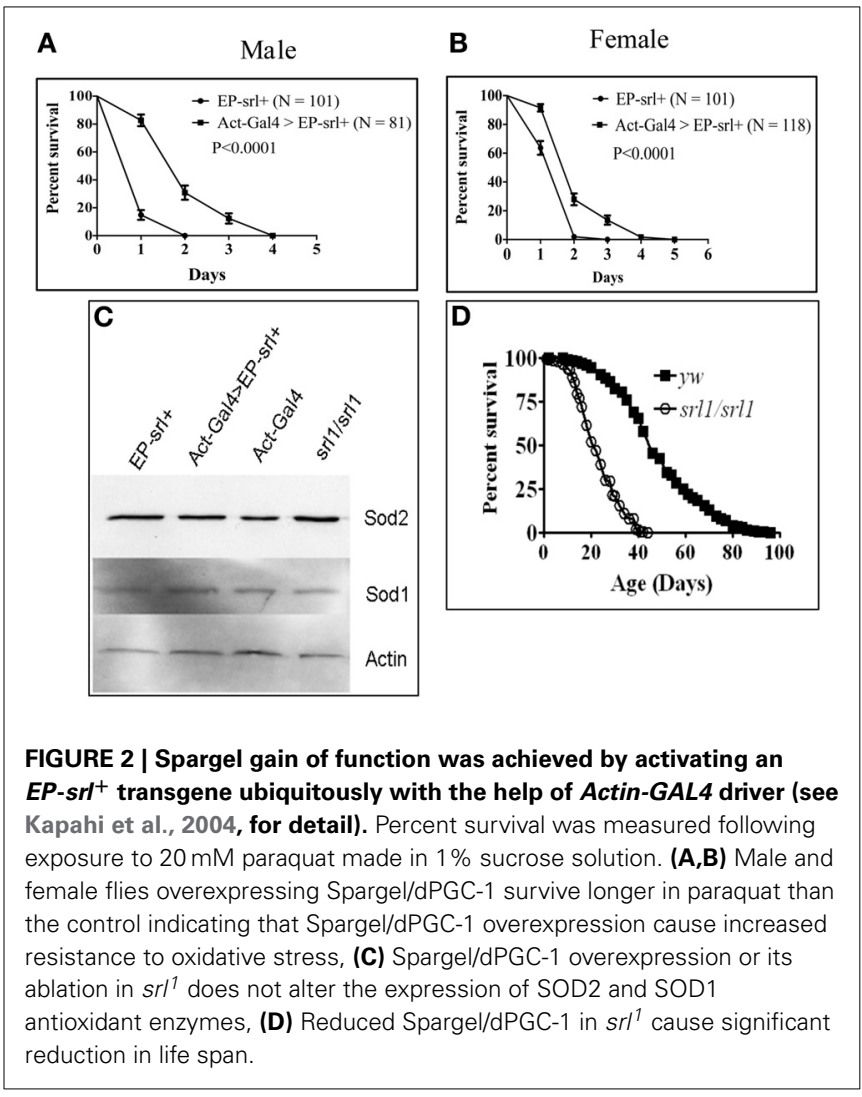

since it is involved in the Insulin-TOR signaling (Mukherjee and Duttaroy, 2013), Spargel/dPGC-1 might utilize other stress resistance pathways (which remain to be tested) such as FoxO or Jnk as opposed to antioxidant enzymes. Incidentally, a recent study in Drosophila showed that Wolbachea induced metabolic stress promotes mitogenesis through activation of Spargel/dPGC-1 (Chen et al., 2012). Clearly, more needs to be done to understand the relationship between Spargel/dPGC-1 and stress resistance.

\section{GROWTH, LONGEVITY, AND AGING}

Insulin-TOR signaling pathway is involved in systemic regulation of growth, longevity and aging in all taxa (Hietakangas and Cohen, 2009; Partridge et al., 2011). In the absence of Insulin, PGC-1 expression is elevated in liver and gluconeogenesis is initiated (Puigserver et al., 2003). Therefore liver-specific knock down of PGC-1 shows higher insulin sensitivity in mice (Koo et al., 2004). On the contrary, absence of PGC-1 in skeletal muscles imposes no effect on insulin sensitivity (Zechner et al., 2010). Thus one can assume that the metabolic role of PGC-1 and its relationship with Insulin/TOR signaling became more tissue specific during the course of evolution.

The requirement of Spargel/dPGC-1 in the Insulin-TOR signaling pathway (Tiefenbock et al., 2010; Mukherjee and Duttaroy, 2013) automatically connects it to the cell growth process. Spargel/dPGC-1 is a terminal effector of this pathway hence reduced spargel expression results in growth retardation with smaller body size and developmental delays, 
though Spargel/dPGC-1 overexpression has no immediate effect on growth (Rera et al., 2011; Mukherjee and Duttaroy, 2013).

With respect to longevity, members of the Insulin-TOR signaling pathway extend life span in C. elegans (Vellai et al., 2003; Kenyon, 2010; Partridge et al., 2011), Drosophila (Clancy et al., 2001; Tatar et al., 2001; Kapahi et al., 2004) and mice (Fontana et al., 2010; Kenyon, 2010) by delaying the aging process. Mutant forms of Insulin receptor (InR), the Insulin receptor substrate chico and TOR all extend longevity, whereas for FoxO lifespan extension happens through its overexpression. Spargel/dPGC1's action on longevity is apparent as reduced Spargel/dPGC-1 expression cause significant shortening of life span (Figure 2D). Reduced ROS production and stem cell over proliferation were cited as two major reasons for Spargel/dPGC-1 mediated extension of lifespan (Rera et al., 2011), though the gain of function effects are specific to the intestinal stem cells as opposed to the Insulin and TOR mediated lifespan extensions, which are largely ubiquitous. So, research on Drosophila is pioneering for understanding the aging aspects of the PGC-1 group of proteins, and we are anxiously awaiting studies on how the mammalian PGC-1 protein influences aging.

To summarize, the effects of Spargel/dPGC-1 on growth and survival are positive, like all the other members of the Insulin/TOR signaling pathway (Mukherjee and Duttaroy, 2013). So, spargel/dPGC-1 loss of function is lethal (Duttaroy et al., in preparation), spargel/dPGC-1 hypomorphs have a much shorter life span (Figure 2D) where as ubiquitous gain of Spargel/dPGC-1 function does not extend lifespan (Rera et al., 2011). Thus, Spargel/dPGC-1 completes the function of InsulinTOR pathway leading to survival.

\section{IS Spargel/dPGC-1 ESSENTIAL FOR FEMALE FERTILITY?}

Reduced Spargel/dPGC-1 expression causes only a few viable adults to appear from $s r l^{1}$ homozygous mothers, indicating that fecundity is seriously compromised in $s r l^{1}$ hypomorphic females though the fertility of $s r l^{1}$ males remain unchanged (Table 1). The growth retardation effect of $s l^{1}$ could be the reason for the appearance of dysgenic ovaries, which carry about half the number of ovarioles compared to the wild type (Figures 3A,B). A simple time course analysis of oogenesis revealed that $s r l^{1}$ ovaries develop slowly. In $24 \mathrm{~h}$ post eclosion (PE) wild type ovarioles reach up to stage $10 / 11$ whereas ovarioles in $\mathrm{srl}^{1}$ are lagging behind in stage $6 / 7$. By $48 \mathrm{~h}$ mature oocytes appear in the wild type ovarioles, whereas most $s l^{1}$ ovarioles are observed around stage 10 during this time (Figure 3C). Efforts are underway to pin down the requirement of Spargel/dPGC-1 during oogenesis through its selective ablation in the ovaries. Interestingly,

\section{Table 1 | Fertility of $\left.s r\right|^{1}$ females.}

\section{Genotype}

Fecundity (\# of adults)

y w 361

$y w ; s r l^{1}$ female $X y w$ male 8

$y w ; s r l^{1}$ male $X y w$ female 296

$y w ; s r l^{1}$ female $X y w, s r l^{1}$ male decreased female fecundity results from oogenesis defects in $I n R$ and chico mutants (Tatar et al., 2001; Partridge et al., 2011). These observations suggested a correlation between Insulin signaling and reproductive aging. Incidentally, the effect of $s r l^{1}$ mutant on
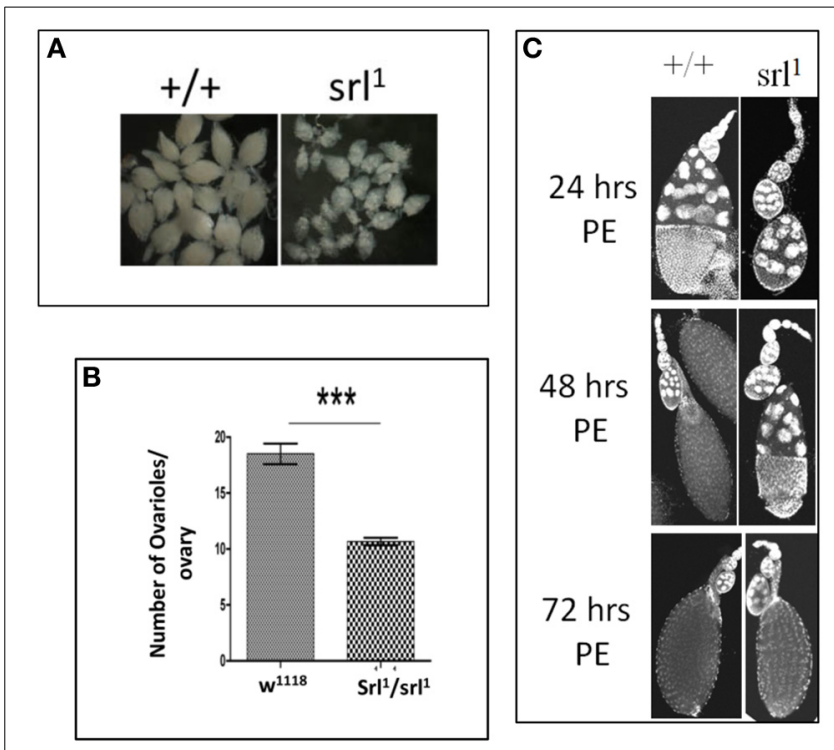

FIGURE 3 | Possible requirement of Spargel/dPGC-1 during oogenesis. (A) Mature ovaries from spargel hypomorph $\mathrm{srl}^{1}$ females are much smaller in size with respect to the control ovaries of same age, (B) Quantification of number of ovarioles suggest $\mathrm{srl}^{1}$ ovaries carry about $40 \%$ less number of ovarioles with respect to the wild type ovaries. (C) Time course analysis of oogenesis shows $\mathrm{Srl}^{1}$ ovaries matures more slowly in comparison to the wild type ovaries (PE, post eclosion). ${ }^{* * *} P<0.001$.

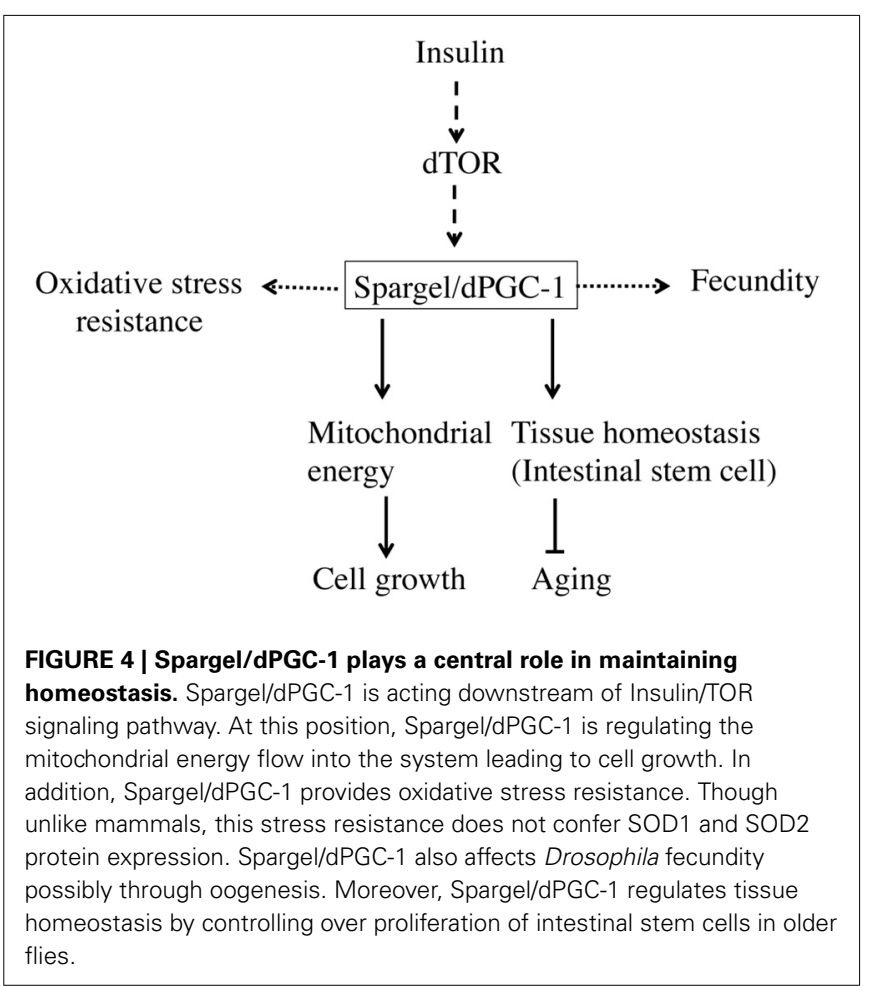


female fecundity and oogenesis appears to suggest that Spargel could be important for reproductive aging.

In summary, the PGC-1 group of proteins retained many important biological functions between vertebrates and invertebrates, though many are still unknown. The overarching hypothesis of this article is that Spargel/dPGC-1 can pave the way to uncover newer biological functions, which can be tested in mammalian PGC-1 (Figure 4). Given the amount of functional overlaps already existing between PGC-1 and Spargel/dPGC1 , some of these similarities may be of immediate interest, including the role of the PGC-1 group of proteins on transcription and RNA processing and finding PGC-1 interacting proteins involved in growth and metabolism. Available genetic tools and genomic reagents in Drosophila should come in handy for exploring the functionality of this omnipotent transcription co-activator.

\section{ACKNOWLEDGMENTS}

We thank the Drosophila Stock Center in Bloomington, Indiana for the fly stocks. Thanks are due to Dr. Nathan Smith for careful reading of the manuscript.

\section{REFERENCES}

Austin, S., and St-Pierre, J. (2012). PGClalpha and mitochondrial metabolismemerging concepts and relevance in ageing and neurodegenerative disorders. $J$. Cell Sci. 125, 4963-4971. doi: 10.1242/jcs.113662

Bugger, H., Guzman, C., Zechner, C., Palmeri, M., Russell, K. S., and Russell, R. R. 3rd. (2011). Uncoupling protein downregulation in doxorubicin-induced heart failure improves mitochondrial coupling but increases reactive oxygen species generation. Cancer Chemother. Pharmacol. 67, 1381-1388. doi: 10.1007/s00280010-1441-7

Chen, S., Oliveira, M. T., Sanz, A., Kemppainen, E., Fukuoh, A., Schlicht, B., et al. (2012). A cytoplasmic suppressor of a nuclear mutation affecting mitochondrial functions in Drosophila. Genetics 192, 483-493. doi: 10.1534/genetics.112.143719

Clancy, D. J., Gems, D., Harshman, L. G., Oldham, S., Stocker, H., Hafen, E., et al. (2001). Extension of life-span by loss of CHICO, a Drosophila insulin receptor substrate protein. Science 292, 104-106. doi: 10.1126/science.10 57991

Coppola, G., Marmolino, D., Lu, D., Wang, Q., Cnop, M., Rai, M., et al. (2009). Functional genomic analysis of frataxin deficiency reveals tissue-specific alterations and identifies the PPARgamma pathway as a therapeutic target in Friedreich's ataxia. Hum. Mol. Genet. 18, 2452-2461. doi: 10.1093/hmg/ ddp 183

Fontana, L., Partridge, L., and Longo, V. D. (2010). Extending healthy life span-from yeast to humans. Science 328, 321-326. doi: 10.1126/science.117 2539

Gershman, B., Puig, O., Hang, L., Peitzsch, R. M., Tatar, M., and Garofalo, R. S. (2007). High-resolution dynamics of the transcriptional response to nutrition in Drosophila: a key role for dFOXO. Physiol. Genomics 29, 24-34. doi: 10.1152/physiolgenomics.00061.2006

Handschin, C., and Spiegelman, B. M. (2006). Peroxisome proliferator-activated receptor gamma coactivator 1 coactivators, energy homeostasis, and metabolism. Endocr. Rev. 27, 728-735. doi: 10.1210/er. 2006-0037

Hietakangas, V., and Cohen, S. M. (2009). Regulation of tissue growth through nutrient sensing. Annu. Rev. Genet. 43, 389-410. doi: 10.1146/annurev-genet102108-134815

Kapahi, P., Zid, B. M., Harper, T., Koslover, D., Sapin, V., and Benzer, S. (2004). Regulation of lifespan in Drosophila by modulation of genes in the TOR signaling pathway. Curr. Biol. 14, 885-890. doi: 10.1016/j.cub.2004. 03.059

Kenyon, C. J. (2010). The genetics of ageing. Nature 464, 504-512. doi: 10.1038/nature08980
Koo, S. H., Satoh, H., Herzig, S., Lee, C. H., Hedrick, S., Kulkarni, R., et al. (2004). PGC-1 promotes insulin resistance in liver through PPARalpha-dependent induction of TRB-3. Nat. Med. 10, 530-534. doi: 10.1038/ nm1044

Lin, J., Handschin, C., and Spiegelman, B. M. (2005). Metabolic control through the PGC-1 family of transcription coactivators. Cell Metab. 1, 361-370. doi: 10.1016/j.cmet.2005.05.004

Liu, C., and Lin, J. D. (2011). PGC-1 coactivators in the control of energy metabolism. Acta Biochim. Biophys. Sin. (Shanghai) 43, 248-257. doi: 10.1093/abbs/gmr007

Matsuda, S., Harries, J. C., Viskaduraki, M., Troke, P. J., Kindle, K. B., Ryan, C., et al. (2004). A conserved alpha-helical motif mediates the binding of diverse nuclear proteins to the SRC1 interaction domain of CBP. J. Biol. Chem. 279, 14055-14064. doi: 10.1074/jbc.M310188200

Monsalve, M., Wu, Z., Adelmant, G., Puigserver, P., Fan, M., and Spiegelman, B. M. (2000). Direct coupling of transcription and mRNA processing through the thermogenic coactivator PGC-1. Mol. Cell 6, 307-316. doi: 10.1016/S10972765(00)00031-9

Mukherjee, S., and Duttaroy, A. (2013). Spargel/dPGC-1 is a new downstream effector in the insulin-TOR signaling pathway in Drosophila. Genetics 195, 433-441. doi: 10.1534/genetics.113.154583

Partridge, L., Alic, N., Bjedov, I., and Piper, M. D. (2011). Ageing in Drosophila: the role of the insulin/Igf and TOR signalling network. Exp. Gerontol. 46, 376-381. doi: 10.1016/j.exger.2010.09.003

Puigserver, P., Rhee, J., Donovan, J., Walkey, C. J., Yoon, J. C., Oriente, F., et al. (2003). Insulin-regulated hepatic gluconeogenesis through FOXO1PGC-1alpha interaction. Nature 423, 550-555. doi: 10.1038/nature 01667

Puigserver, P., and Spiegelman, B. M. (2003). Peroxisome proliferator-activated receptor-gamma coactivator 1 alpha (PGC-1 alpha): transcriptional coactivator and metabolic regulator. Endocr. Rev. 24, 78-90. doi: 10.1210/er. 2002-0012

Puigserver, P., Wu, Z., Park, C. W., Graves, R., Wright, M., and Spiegelman, B. M. (1998). A cold-inducible coactivator of nuclear receptors linked to adaptive thermogenesis. Cell 92, 829-839. doi: 10.1016/S0092-8674(00)81410-5

Rera, M., Bahadorani, S., Cho, J., Koehler, C. L., Ulgherait, M., Hur, J. H., et al. (2011). Modulation of longevity and tissue homeostasis by the Drosophila PGC-1 homolog. Cell Metab. 14, 623-634. doi: 10.1016/j.cmet.2011.09.013

St-Pierre, J., Drori, S., Uldry, M., Silvaggi, J. M., Rhee, J., Jager, S., et al. (2006). Suppression of reactive oxygen species and neurodegeneration by the PGC-1 transcriptional coactivators. Cell 127, 397-408. doi: 10.1016/j.cell.2006. 09.024

Takano, A., Usui, I., Haruta, T., Kawahara, J., Uno, T., Iwata, M., et al. (2001). Mammalian target of rapamycin pathway regulates insulin signaling via subcellular redistribution of insulin receptor substrate 1 and integrates nutritional signals and metabolic signals of insulin. Mol. Cell. Biol. 21, 5050-5062. doi: 10.1128/MCB.21.15.5050-5062.2001

Tatar, M., Kopelman, A., Epstein, D., Tu, M. P., Yin, C. M., and Garofalo, R. S. (2001). A mutant Drosophila insulin receptor homolog that extends life-span and impairs neuroendocrine function. Science 292, 107-110. doi: 10.1126/science. 1057987

Tiefenbock, S. K., Baltzer, C., Egli, N. A., and Frei, C. (2010). The Drosophila PGC1 homologue Spargel coordinates mitochondrial activity to insulin signalling. EMBO J. 29, 171-183. doi: 10.1038/emboi.2009.330

van der Linde, K., and Houle, D. (2008). A supertree analysis and literature review of the genus Drosophila and closely related genera (Diptera, Drosophilidae). Insect. Syst. Evol. 39, 241-267. doi: 10.1163/187631208788784237

Vellai, T., Takacs-Vellai, K., Zhang, Y., Kovacs, A. L., Orosz, L., and Muller, F. (2003). Genetics: influence of TOR kinase on lifespan in C. elegans. Nature 426, 620. doi: $10.1038 / 426620$ a

Wang, J., Li, Y., Zhang, M., Liu, Z., Wu, C., Yuan, H., et al. (2007). A zinc finger HIT domain-containing protein, ZNHIT-1, interacts with orphan nuclear hormone receptor Rev-erbbeta and removes Rev-erbbeta-induced inhibition of apoCIII transcription. FEBS J. 274, 5370-5381. doi: 10.1111/j.1742-4658.2007. 06062.x

Zechner, C., Lai, L., Zechner, J. F., Geng, T., Yan, Z., Rumsey, J. W., et al. (2010). Total skeletal muscle PGC-1 deficiency uncouples mitochondrial derangements from fiber type determination and insulin sensitivity. Cell Metab. 12, 633-642. doi: 10.1016/j.cmet.2010.11.008 
Conflict of Interest Statement: The authors declare that the research was conducted in the absence of any commercial or financial relationships that could be construed as a potential conflict of interest.

Received: 03 May 2014; accepted: 22 June 2014; published online: 10 July 2014. Citation: Mukherjee S, Basar MA, Davis C and Duttaroy A (2014) Emerging functional similarities and divergences between Drosophila Spargel/dPGC-1 and mammalian PGC-1 protein. Front. Genet. 5:216. doi: 10.3389/fgene.2014.00216
This article was submitted to Genetics of Aging, a section of the journal Frontiers in Genetics.

Copyright (C) 2014 Mukherjee, Basar, Davis and Duttaroy. This is an open-access article distributed under the terms of the Creative Commons Attribution License (CC BY). The use, distribution or reproduction in other forums is permitted, provided the original author(s) or licensor are credited and that the original publication in this journal is cited, in accordance with accepted academic practice. No use, distribution or reproduction is permitted which does not comply with these terms. 